\title{
Hipertensión arterial en entidades endocrinas
}

\section{Arturo Orrego M.}

Médico Internista Endocrinólogo, Miembro Honorario de la Asociación Colombiana de Endocrinología, Diabetes y

Metabolismo.

Fecha de recepción: 10/12/2017

Fecha de aceptación: 31/01/2018

\section{Generalidades}

$\mathrm{L}$ a hipertensión se presenta en una tercera parte de las personas adultas en Estados Unidos ${ }^{(1-3)}$. La gran mayoría de estos hipertensos son de origen esencial o idiopáticos, pero se ha identificado que aproximadamente el $15 \%$ presentan hipertensión secundaria ${ }^{(4,5)}$. Más del $50 \%$ de los niños que presentan hipertensión son portadores de una hipertensión secundaria ${ }^{(6)}$. En los individuos hipertensos mayores de 40 años, la hipertensión arterial es de origen secundario en un $30 \%$ de los $\operatorname{casos}^{(7)}$. Las causas secundarias de hipertensión arterial son de origen renal, compromiso parenquimatoso de los riñones y de origen endocrino. La hipertensión arterial puede ser una de las primeras manifestaciones de al menos 15 endocrinopatías (tabla 1). Un diagnóstico acertado y temprano de una hipertensión endocrina puede dar la garantía al médico tratante de la posibilidad de conseguir con cirugía una curación de la hipertensión o una respuesta clínica óptima con la terapia farmacológica adecuada y específica ${ }^{(8)}$. Existe suficiente evidencia de que se debe considerar el diagnóstico de hiperaldosteronismo primario en la mayoría de los casos con hipertensión arterial ${ }^{(9)}$. En este artículo trataremos de revisar las diferentes presentaciones de las endocrinopatías acompañadas de hipertensión arterial, su prevalencia, presentación clínica y su diagnóstico, por medio del laboratorio, con la utilización de pruebas presuntivas y específicas.

\section{Feocromocitoma y paraganglioma}

\section{Generalidades y prevalencia}

El feocromocitoma y el paraganglioma son tumores neuroendocrinos de células cromafinas que característicamente producen catecolaminas y que se originan, el $80 \%$ en la médula adrenal y el $15 \%$ al $20 \%$ en los ganglios paravertebrales de la cadena simpática. Los paragangliomas originados en la nuca y en la base del cráneo por lo general son hormonalmente inactivos, excepto algunos que producen dopamina. La prevalencia de los paragangliomas es muy baja, 1,5 por cada 10.000 personas. La prevalencia aumenta significativamente (20 a 60 por 10.000 pacientes) en los portadores de hipertensión arterial $^{(10,11)}$. En ciertos grupos de pacientes, la prevalencia es mucho mayor (500 casos por cada 10.000 individuos), pacientes en los cuales se encuentra un incidentaloma adrenal ${ }^{(12)}$. Con frecuencia no se diagnostican en vida los paragangliomas por su rareza, ya que la prevalencia de estos tumores en las autopsias es de 5 por cada 1.000 individuos $^{(13,14)}$.

Los paragangliomas pueden ser adrenérgicos y noradrenérgicos ${ }^{(15,16)}$. Los tumores adrenérgicos están localizados en la médula adrenal y generalmente secretan epinefrina, meta-

Tabla 1. Entidades endocrinas con hipertensión

\section{Causas de origen adrenal}

1. Feocromocitoma y paraganglioma simpático

2. Aldosteronismo primario

3. Hiperdeoxicorticosteroidismo

a. Hiperplasia adrenal congénita

Deficiencia de la $11 \beta$ - hidroxilasa

Deficiencia de la $17 \beta$ - hidroxilasa

b. Tumores productores de desoxicorticosterona

c. Resistencia primaria al cortisol

4. Síndrome de Cushing

Aparentemente exceso de mineralocorticoides,

deficiencia de $11 \beta$-hidroxiesteroide deshidrogenasa.

1. Genética

2. Adquirida

a. Ingestión de licorice o de carbenoxolona

b. Síndrome de Cushing

Dependiente de la Paratohormona

1. Hiperparatiroidismo

Dependiente de causas hipofisiarias

1. Acromegalia

2. Síndrome de Cushing

Hiperaldosteronismo secundario

1. Hipertensión renovascular

Causas dependientes de tiroides

Hipotiroidismo

Hipertiroidismo

Apnea obstructiva del sueño 
nefrina (metabolito mayor de la epinefrina) y cantidades variables de norepinefrina. Los tumores noradrenérgicos están localizados ya sea en la médula adrenal o son extradrenales y producen principal o exclusivamente norepinefrina y normetanefrina (metabolito mayor de la norepinefrina). La ausencia de secreción de la epinefrina en estos tumores se debe a la ausencia de una enzima la feniletanolamina-N-metiltransferasa en los tumores extradrenales ${ }^{(15,16)}$. Genéticamente, los tumores noradrenérgicos tienen tendencia mayor a estar asociados con mutaciones del tipo Hippel-Lindau y de la succinato deshidrogenasa, en cambio los tumores adrenérgicos se encuentran más frecuentemente acompañados de mutaciones de ciertas cinasas que dan origen a neoplasias endocrinas múltiples tipo 2 y a la neurofibromatosis tipo 1 . Como rasgo característico, los tumores adrenérgicos tienen tendencia a producir síntomas paroxísticos más frecuentemente que los tumores noradrenérgicos ${ }^{(17,18)}$.

\section{Manifestaciones clínicas}

Las manifestaciones clínicas de los paragangliomas varían extensamente en cuanto a su presentación, pueden ser asintomáticos o con manifestaciones mínimas o dar origen a síntomas catastróficos que amenazan la vida de los pacientes ${ }^{(10,19)}$.

Puede afirmarse que uno de cada 10 pacientes es asintomático; este grupo sin síntomas es más aparente en los pacientes con un incidentaloma adrenal o en aquellos investigados durante un tamizaje familiar para feocromocitoma.

Como típico, la triada clásica de cefalea opresiva, sudoración profusa y palpitaciones que ocurren en crisis, de varios minutos a una hora de duración, sugiere el diagnóstico de paraganglioma. Entre las crisis de cefalea existen períodos asintomáticos. La frecuencia de las crisis puede ser de varias al día a unas pocas por mes, de ocurrencia espontánea o provocada por diferentes estímulos químicos o físicos, tales como la anestesia, la micción y ciertos medicamentos (inhibidores betaadrenérgicos, antidepresivos tricíclicos y los glucocorti-

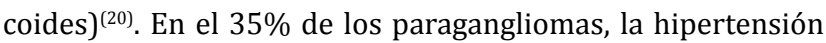
arterial se presenta en crisis paroxísticas. En otros, las crisis hipertensivas pueden sumarse a la hipertensión persistente de los pacientes. Se cree que estas crisis hipertensivas pueden aumentar la prevalencia de las emergencias cardiovasculares, tales como el infarto del miocardio, la hemorragia cerebral y la falla miocárdica ${ }^{(20)}$.

\section{Tamizaje en los pacientes sospechosos}

Para el tamizaje en busca de paragangliomas debe tenerse en cuenta la presencia del exceso de secreción de catecolaminas. La presencia de una hipertensión paroxística debe estimular al estudio de paraganglioma con pruebas bioquímicas, independiente del grado de hipertensión. En los pacientes en quienes se encuentra un incidentaloma adrenal debe investigarse la presencia de paraganglioma por medios bioquími- $\cos ^{(21)}$. Se debe investigar bioquímicamente a quien recientemente se le descubrió un paraganglioma ${ }^{(22)}$.

\section{Pruebas de tamizaje}

Como pruebas iniciales para el diagnóstico de los paragangliomas debe ordenarse la medición de metanefrinas libres o fraccionadas ${ }^{(22)}$. Debido a la alta sensibilidad de estas pruebas, valores normales hacen muy improbable el diagnóstico de paraganglioma, a menos que los tumores sean muy pequeños.

La precisión de estas pruebas es similar en sensibilidad si se mide en plasma o en orina de 24 horas, especialmente si para medirla se utilizan la cromatografía líquida y la espectrometría de masas ${ }^{(20)}$. La explicación de la alta sensibilidad de estas mediciones es la secreción continuada de metanefrinas por el paraganglioma que contrasta con la secreción episódica de las catecolaminas.

Otras pruebas bioquímicas, tales como las catecolaminas plasmáticas o urinarias, el ácido vanilmandélico urinario y la cromogranina A tienen menor valor que las metanefrinas fraccionadas plasmáticas o urinarias en el diagnóstico del paraganglioma.

\section{Pacientes que deben investigarse para paraganglioma}

En la tabla 2 y en la figura 1 se dan indicaciones de a quiénes deben ordenarse pruebas bioquímicas para investigar la presencia de feocromocitomas o paragangliomas.

Tabla 2. Cuándo se deben ordenar pruebas bioquímicas en busca del feocromocitoma o paraganglioma

\section{Indicaciones}

En presencia de signos o síntomas sugestivos de exceso de catecolaminas

Respuesta paradójica de la presión arterial a drogas, cirugía o anestesia

Hipertensión arterial resistente

Descubrimiento de un incidentaloma adrenal

En busca del diagnóstico recurrente de feocromocitoma o paraganglioma

Predisposición hereditaria para el feocromocitoma o paraganglioma

Hechos que sugieren un síndrome hereditario relacionado con un feocromocitoma

Los clínicos deben tomar varias precauciones y conocer que ciertos estados, por un aumento de la actividad simpática, tales como la hipoglucemia, la insuficiencia cardiaca, la falla renal, pueden dar origen a cifras elevadas de catecolaminas o metanefrinas. Para minimizar el riesgo de falsos positivos en la medición de las metanefrinas libres en plasma, la muestra se debe tomar por catéter después de un reposo en posición 
Figura 1. Manifestaciones que hacen sospechar feocromocitoma o hiperaldosteronismo primario que deben sugerir investigación de la entidad

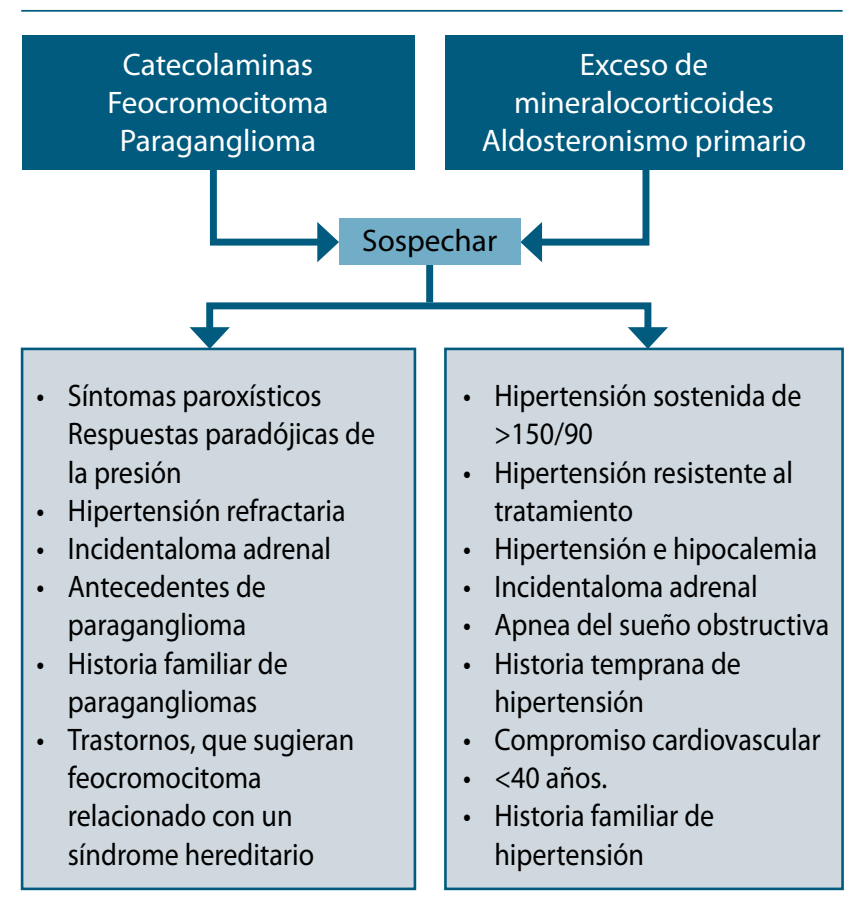

supina durante 30 minutos. Si no es posible tomar la muestra de sangre, la medición de las metanefrinas fraccionadas en orina de 24 horas es un método alternativo de alto valor. Para la medición de metanefrinas plasmáticas libres es conveniente tomar la muestra en ayunas para evitar la presencia de un metabolito de la dopamina, la metoxitiramina que puede aumentar durante los periodos de no ayuno ${ }^{(20)}$. La elevación de ese metabolito sugiere la presencia de un tumor extradrenal, localizado en la nuca o en el cuello ${ }^{(23)}$. Varias drogas pueden ser fuente de falsos positivos por interferencias (tabla 3). La medición de las metanefrinas es mejor realizarla con cromatografía líquida con espectrometría por ser más específicas por las menores interferencias por drogas ${ }^{(20)}$, utilizando tubo heparinizado, el cual debe colocarse diariamente dentro del recipiente con hielo, para prevenir la oxidación.

\section{Valoración de los resultados de las pruebas}

Si se desea obtener resultados precisos, cada laboratorio debe tener sus propios valores de referencia de las metanefrinas fraccionadas urinarias y de las libres de plasma, en las cuales se debe tener en cuenta la edad y el sexo de los pacientes. Debe recordarse que los niveles de normalidad de las metanefrinas libres en plasma se incrementan con la edad ${ }^{(20)}$.

Si los niveles de metanefrinas libres en plasma o las metanefrinas fraccionadas en orina en un paciente sintomático son normales, puede excluirse el diagnóstico de paraganglioma, siempre y cuando la recolección de orina haya sido completa y las muestras de sangre hayan sido tomadas dentro de las normas especificadas. En un paciente asintomático, un único resultado normal no excluye un paraganglioma; es mejor en estos casos esperar y ordenar nuevos exámenes más tarde.

La presencia de unos niveles de metanefrinas plasmáticas libres, mayores de más de tres veces los límites superiores normales y de las metanefrinas fraccionadas en orina por encima de más de dos veces los límites normales, sugieren la presencia de un paraganglioma. En estos casos, el riesgo de un falso positivo es muy bajo. El médico puede iniciar la búsqueda de la localización del paraganglioma por medios imaginológicos. Debe recordarse que el $25 \%$ de los pacientes puede tener niveles por fuera de lo normal pero no muy elevados, lo que dificulta la decisión para definir si en realidad existe un paraganglioma. En los pacientes hospitalizados en cuidados intensivos es frecuente encontrar falsos positivos, por lo cual nunca deben investigarse pacientes para paragangliomas, cuando están en estado crítico $^{(20)}$.

En pacientes con niveles de metanefrinas libres o fraccionadas discretos, el clínico debe descartar si esos resultados pueden deberse a condición de estrés, o a otros estados, capaces de aumentar la actividad simpática y no a un paraganglioma.

Existen otras condiciones con discreta elevación de las metanefrinas, por ejemplo, la obtención de la muestra de sangre en paciente sin reposo previo, acostado por mínimo 30 minutos antes. Antes de obtener la muestra de sangre o de la recolección de orina, es imperativo descontinuar las drogas capaces de producir falsos positivos, por ejemplo, los antidepresivos tricíclicos (tabla 3). Aconsejan medir simultáneamente las metanefrinas fraccionadas en orina y la cromogranina A plasmática para descartar los niveles plasmáticos de metanefrinas falsamente elevados ${ }^{(24)}$. Como digno de mencionar es la supresión con clonidina para diferenciar una elevación real tumoral de las normetanefrinas de un incremento falsamente positivo por activación simpática ${ }^{(25)}$.

\section{Aldosteronismo primario}

En esta entidad, la producción de aldosterona excede los límites normales y permanece fuera del control del sistema renina-angiotensina II. La producción de aldosterona en exceso, sin regulación, produce un aumento de la reabsorción del sodio a nivel del nefrón distal, dando origen a hipertensión arterial y a la supresión del sistema renina-angiotensina II. La pérdida urinaria de potasio y de iones de hidrógeno, en intercambio con sodio, en el nefrón distal, puede dar origen a hipocaliemia y alcalosis metabólica ${ }^{(26,27)}$.

\section{Prevalencia y presentación clínica}

En un principio se creyó que el hiperaldosteronismo primario era una entidad poco común, en cambio en estos momentos esta entidad es considerada como la más común, tratable y potencialmente curable de los estados acompañados 
Tabla 3. Medicamentos que causan falsos positivos con las pruebas bioquímicas en los casos de feocromocitoma o paraganglioma simpático

\begin{tabular}{l|c|c|c|c}
\hline \multirow{2}{*}{\multicolumn{1}{c|}{ Medicamento }} & \multicolumn{2}{|c|}{ Plasma } & \multicolumn{2}{c}{ Orina } \\
\cline { 2 - 5 } & NMN & MN & NMN & MN \\
\hline $\begin{array}{l}\text { Antidepresivos } \\
\text { tricíclicos }\end{array}$ & $\uparrow \uparrow$ & - & $\uparrow \uparrow$ & - \\
\hline Antipsicóticos & $\uparrow \uparrow$ & & $\uparrow \uparrow$ & - \\
\hline Buspirona & $\uparrow$ & $\uparrow \uparrow$ & $\uparrow$ & $\uparrow \uparrow$ \\
\hline Inhibidores MAO & $\uparrow \uparrow$ & $\uparrow \uparrow$ & $\uparrow \uparrow$ & $\uparrow \uparrow$ \\
\hline Simpaticomiméticos & $\uparrow$ & $\uparrow$ & $\uparrow$ & $\uparrow$ \\
\hline Cocaína & $\uparrow \uparrow$ & $\uparrow$ & $\uparrow \uparrow$ & $\uparrow$ \\
\hline Levodopa & $\uparrow$ & $\uparrow$ & $\uparrow \uparrow$ & $\uparrow$ \\
\hline Fenoxibenzamina & $\uparrow \uparrow$ & - & $\uparrow \uparrow$ & - \\
\hline Acetaminofén & $\uparrow \uparrow$ & - & $\uparrow \uparrow$ & - \\
\hline Labetalol & - & - & $\uparrow \uparrow$ & $\uparrow \uparrow$ \\
\hline Sotalol & - & - & $\uparrow \uparrow$ & $\uparrow \uparrow$ \\
\hline Alfa-metildopa & $\uparrow \uparrow$ & - & $\uparrow \uparrow$ & - \\
\hline Sulfasalazina & $\uparrow \uparrow$ & - & $\uparrow \uparrow$ & - \\
\hline
\end{tabular}

- No cambio; MN: metanefrinas, NMN, normetanefrinas. Las interferencias farmacodinámicas afectan todos los métodos analíticos.

de hipertensión arterial; está presente en el 5\% al 10\% de los pacientes hipertensos. En los pacientes con hipertensión arterial resistente al tratamiento, la frecuencia del hiperaldosteronismo primario alcanza las cifras de un $20 \%{ }^{(27,28,29)}$. La mayoría de los hiperaldosteronismos primarios se diagnostican entre la tercera y sexta década ${ }^{(20)}$. En el momento actual, se ha encontrado que la mayoría de los pacientes con hiperaldosteronismo primario son normocaliémicos.

\section{Hallazgos clínicos}

La hipertensión arterial se presenta en la mayoría de los pacientes con hiperaldosteronismo primario y puede ser mínima o intensa, pero raramente maligna ${ }^{(20)}$. El grado de hipertensión arterial no diferencia el hiperaldosteronismo debido a hiperplasia adrenal bilateral del originado en un adenoma ${ }^{(30)}$. En el hiperaldosteronismo familiar tipo 1, la presentación de la hipertensión arterial puede ser tardía, especialmente en mujeres, pero también puede ser de comienzo temprano y tan grave que puede producir la muerte, debido a compromiso cerebral por hemorragia. Los hiperaldosteronismos familiares tipo I y II presentan diferentes y variados fenotipos, con la presencia de presión arterial normal, en algunos ${ }^{(20)}$.

Menos de un cuarto de los pacientes en quienes se diagnosticó un hiperaldosteronismo primario y menos de $50 \%$ de los portadores de adenomas productores de hiperaldosteronismo primario fueron hipocaliémicos ${ }^{(20)}$, lo que sugiere que un hiperaldosteronismo primario normocaliémico puede ser imposible de diferenciar frente a una hipertensión arterial no endocrina, sí no se mide renina y aldosterona. Cuando se presenta hipocaliemia, puede observarse en el paciente nicturia, poliuria, debilidad muscular, parestesias, calambres y palpitaciones. La prevalencia de apnea del sueño obstructiva se incrementa en pacientes con hiperaldosteronismo primario, y mejora con el control del exceso de aldosterona ${ }^{(31)}$.

Durante el embarazo, la hipertensión y los síntomas de hiperaldosteronismo primario pueden mejorar o agravarse. La mejoría parece deberse al exceso de la progesterona placentaria circulante, por su efecto antimineralocorticoide que antagoniza la acción del exceso de aldosterona ${ }^{(20)}$. En los casos en los cuales se agrava el hiperaldosteronismo primario durante el embarazo se puede deber al exceso de gonadotropina coriónica humana circulante y a la activación de los receptores de la hormona liberadora de gonadotropinas y de la hormona luteínica.

\section{Tamizaje de los pacientes sospechosos}

El hiperaldosteronismo primario es una entidad que debe investigarse en la mayoría de los pacientes hipertensos. Esta idea debe aplicarse en los hipertensos, ya que la hipertensión arterial del hiperaldosteronismo responde bien a un tratamiento específico debido al exceso de aldosterona circulante. La resección laparoscópica unilateral de un adenoma único adrenal cura la hipertensión en el 50\% a $60 \%$ de los casos y mejora significativamente a los pacientes restantes ${ }^{(20,27,31-34)}$. Para los pacientes no operados, la administración de drogas que antagonicen las acciones de la aldosterona, como la espironolactona, la eplerenona y el amiloride, son capaces de controlar significativamente la hipertensión $\operatorname{arterial}^{(20)}$. Se ha demostrado que el exceso de aldosterona circulante produce directamente inflamación, remodelación y fibrosis en los sistemas cardiovascular y renal, lo que lleva a efectos metabólicos adversos, parcialmente independientes del grado de hipertensión arterial ${ }^{(20)}$. Como efecto de estos daños cardiovasculares y renales, en el hiperaldosteronismo primario son más frecuentes las muertes por arritmias cardiacas, hemorragias cerebrales e insuficiencia renal que en la hipertensión arterial esencial $^{(20)}$.

La Sociedad Endocrina, en su guía de práctica clínica, llama la atención en la necesidad de descubrir tempranamente los casos del hiperaldosteronismo primario ${ }^{(9)}$. Según este grupo, deben priorizarse el estudio de los pacientes con alta prevalencia de presentar hiperaldosteronismo primario. Estos incluyen pacientes con presión arterial elevada permanente 2150/100 mmHg; hipertensión $>140 / 90 \mathrm{mmHg}$ resistentes a tres diferentes drogas hipotensoras convencionales con inclusión de un diurético; pacientes con hipertensión controlada $<140 / 90 \mathrm{mmHg}$, gracias a las administración de cuatro o más hipotensores conocidos; deben estudiarse, además, los pacientes que presentan hipocaliemia espontánea o inducida, hipertensos con incidentaloma adrenal, portadores de apnea 
obstructiva o hipertensos de origen familiar, con una iniciación de hipertensión tempranamente o la presentación de un accidente cerebrovascular, por debajo de los 40 años. Debido a la existencia de hiperaldosteronismo familiar, las guías aconsejan el tamizaje en los familiares hipertensos con primer grado de consanguinidad ${ }^{(20)}$ (figura 1).

\section{Pruebas diagnósticas \\ Potasio plasmático}

Debido a que únicamente el $20 \%$ de los pacientes con hiperaldosteronismo primario son hipocaliémicos, el valor de esta medición es mínimo ${ }^{(20)}$. Sin embargo, la presencia de hipocaliemia espontánea, no provocada con diuréticos, sirve como indicio de la probable existencia de un hiperaldosteronismo primario.

\section{La relación aldosterona/renina (ARR)}

La relación aldosterona/renina es la prueba más útil en el tamizaje del aldosteronismo primario, es más sensible que la hipocaliemia o la medición de la aldosterona plasmática ${ }^{(31)}$. La relación ARR puede dar origen a falsos positivos en mujeres premenopáusicas o en quienes están recibiendo estrógenos en anticonceptivos.

Falsos positivos de la ARR también pueden verse en pacientes con disfunción renal (la hipercaliemia que acompaña la falla renal disminuye la secreción de renina y aumenta la producción de aldosterona) ${ }^{(20)}$; con la edad, la producción de renina desciende lo mismo que en la hipertensión arterial hipercaliémica familiar, también conocida como pseudohipoaldesteronismo tipo II y síndrome de Gordon ${ }^{(20)}$.

Falsos positivos de la ARR también pueden presentarse cuando la secreción de renina es estimulada por restricción de la sal en la dieta, en la hipertensión arterial maligna, en el embarazo, con la administración de diuréticos (incluyendo la espirolactona), la dihidropiridina; de los inhibidores de la enzima convertidora de angiotensina, y los antagonistas de los receptores de la angiotensina II $^{(20)}$.

Los bloqueadores beta, la $\alpha$-metildopa, la clonidina y los esteroides antiinflamatorios pueden suprimir las concentraciones plasmáticas de renina y dar origen a falsos positivos de la $\mathrm{ARR}^{(20)}$ (tabla 3).

Los inhibidores de la recaptación de la serotonina disminuyen los resultados de la ARR.

Antes del estudio del hiperaldosteronismo primario, los clínicos deben suspender los diuréticos mínimo cuatro semanas y otras drogas que interfieran con la medición de la ARR, deben suspenderse dos a cuatro semanas antes. Para evitar el descontrol de la presión arterial con la suspensión de los hipotensores deben administrarse drogas como el verapamilo, con o sin hidralazina y prazocín ${ }^{(20)}$. En los casos en los cuales es imposible suspender la medicación que podría interferir con los resultados, el clínico debe conocer los efectos de las drogas administradas cuando va a interpretar los resultados. Una relación ARR elevada en pacientes que están recibiendo diuréticos, inhibidores de la ECA, bloqueadores de los receptores de la angiotensina o bloqueadores del calcio sugieren con buenas probabilidades la presencia de un hiperaldosteronismo primario, en cambio un resultado normal de esta relación durante la administración de bloqueadores beta hace muy improbable el diagnóstico.

Los clínicos deben corregir la hipocaliemia y liberar la ingestión de sal antes de medir la ARR como un medio de tamizaje y se debería repetir, si el caso así lo indica, antes de decidir si ordenar la prueba de supresión con clonidina.

Aunque las cifras de referencia de la aldosterona pueden variar de laboratorio a laboratorio, una concentración plasmática de $>10 \mathrm{ng} / \mathrm{dL}$, asociada a una renina plasmática de $<1 \mathrm{mg} /$ $\mathrm{mL} / \mathrm{h}$ debe indicar que es necesario confirmar el diagnóstico de hiperaldosteronismo primario. Algunos autores consideran que el clínico debe enviar estos casos a un endocrinólogo.

\section{Hiperdesoxicorticosteronismo}

En la tabla 4 se mencionan los trastornos asociados con el exceso de mineralocorticoides, la desoxicorticosterona (DOC) o el cortisol. Los clínicos deberían considerar estos diagnósticos si los niveles sanguíneos de aldosterona y renina son bajos en un paciente hipertenso e hipocaliémico ${ }^{(20)}$.

\section{Hiperplasia adrenal congénita}

La hiperplasia adrenal congénita agrupa a varias entidades autosómicas recesivas, causadas por defectos enzimáticos en la esteroidogénesis que traen consigo la deficiencia en la secreción de cortisol $^{(35)}$. La falta del fenómeno de retroalimentación por el cortisol, a nivel hipotalámico e hipofisiario, produce exceso en la producción de precursores del cortisol, próximos a las deficiencias enzimáticas.

Aproximadamente el 90\% de las hiperplasias adrenales se deben a deficiencia de la 21-hidroxilasa. Las deficiencias de las $11 \beta$-hidroxilasa o de la $17 \alpha$-hidroxilasa producen hipertensión e hipocaliemia debidas a la hipersecreción del mineralocorticoide DOC, con la disminución de la secreción de la renina y la aldosterona. Estas mutaciones son autosómicas recesivas y son diagnosticadas casi siempre en la niñez. Sin embargo, defectos enzimáticos parciales pueden dar origen a hipertensión en adultos ${ }^{(20)}$.

\section{Deficiencia de la 11ß- hidroxilasa}

La deficiencia de la $11 \beta$ - hidroxilasa causa aproximadamente el $5 \%$ de todos los casos de las hiperplasias adrenales congénitas. La prevalencia en blancos es de 1 por cada 100.000 pacientes $^{(20)}$. La prevalencia es mayor entre judíos sefarditas de Marruecos ${ }^{(20,36)}$. 
Tabla 4. Pruebas relacionadas con la adrenal en pacientes con exceso de mineralocorticoides no mediados por aldosterona

\begin{tabular}{l|c|c|c|c|c|c}
\hline \multicolumn{1}{c|}{ Entidad } & $24 \mathrm{~h} \mathrm{UFC}$ & $\begin{array}{c}\text { Relación urinaria } \\
\text { cortisona/cortisol }\end{array}$ & DOC & 11-deoxicortisol & $\begin{array}{c}\text { Androste- } \\
\text { nediona }\end{array}$ & DHEAS \\
\hline $\begin{array}{l}\text { Deficiencia de la 11ß } \\
\text { hidroxilasa }\end{array}$ & $\downarrow$ & - & $\uparrow \uparrow \uparrow$ & $\uparrow \uparrow \uparrow$ & $\uparrow \uparrow \uparrow$ & $\uparrow \uparrow \uparrow$ \\
\hline $\begin{array}{l}\text { Deficiencia de la } \\
\text { 17a-hidroxilasa }\end{array}$ & $\downarrow$ & - & $\uparrow$ & $\uparrow$ & $\downarrow$ & $\downarrow$ \\
\hline $\begin{array}{l}\text { Tumores productores } \\
\text { de DOC }\end{array}$ & - & - & $\uparrow \uparrow \uparrow$ & - & - & - \\
\hline $\begin{array}{l}\text { Resistencia primaria } \\
\text { al cortisol }\end{array}$ & $\uparrow \uparrow$ & - & $\uparrow$ & $\uparrow$ & $\uparrow$ & $\uparrow$ \\
\hline $\begin{array}{l}\text { Aparente exceso de } \\
\text { mineralocorticoides }\end{array}$ & $\uparrow \uparrow \uparrow \uparrow$ & $\uparrow \uparrow \uparrow \uparrow$ & $\uparrow$ & - & - & - \\
\hline
\end{tabular}

- Negativo; UFC, cortisol urinario libre.

Todos los pacientes tienen niveles bajos o indetectables de aldosterona. DHEAS, Dehidroenpiandrosterona sulfato.

\section{Presentación clínica}

La deficiencia en la conversión de DOC a corticosterona resulta en niveles altos de DOC y $11 \beta$-desoxicortisol, lo que trae consigo la elevación de los andrógenos adrenales. Las niñas con hiperplasia adrenal congénita por este defecto enzimático presentan en la infancia o la niñez, hipertensión arterial, hipocaliemia, acné, hirsutismo y virilización. En cambio, los niños con deficiencia de la 11ß-hidroxilasa presentan hipertensión, hipocaliemia y seudopubertad precoz. El 75\% de los pacientes desarrollan hipertensión arterial leve o moderada.

Se deben investigar niños, adolescentes y adultos jóvenes que presentan hipertensión, hipocaliemia espontánea y niveles bajos de renina y aldosterona ${ }^{(20)}$. Deben investigarse preferencialmente mujeres con virilización y muchachos con seudopubertad precoz.

\section{Estudios bioquímicos}

Deben medirse en sangre DOC, 11-desoxicortisol, androstenediona, testosterona y dehidroepiandrosterona sulfato, todos los cuales deben estar con niveles por encima de lo normal. Si los pacientes con hipertensión e hipocaliemia presentan estos niveles elevados, se deberían ordenar pruebas confirmatorias, incluyendo estudios genéticos ${ }^{(20)}$.

\section{Deficiencia de la $17 \alpha$-hidroxilasa}

Esta entidad es muy rara: menos de 1 en 1.000 .000 de nacidos vivos. La enzima $17 \alpha$-hidroxilasa es indispensable para la síntesis de cortisol y hormonas gonadales ${ }^{(20,36)}$ por lo que los hombres 46XY presentan, ya sea seudohermafroditismo o fenotípicamente lucen como mujeres y las mujeres 46XX presentan amenorrea primaria. Estos casos generalmente no llegan al estudio médico antes de la pubertad.
Los clínicos deben estudiar los niños, los adolescentes y adultos jóvenes que presentan hipertensión e hipocaliemia espontánea y niveles bajos de aldosterona y renina. Se debe sospechar esta mutación preferencialmente en los casos con amenorrea primaria y seudohermafroditismo. Esta mutación es más frecuente entre los alemanes menonitas ${ }^{(20)}$.

Como tamizaje inicial se debe medir en sangre androstenediona, testosterona, dehidroepiandrosterona sulfato, 17-hidroxiprogesterona, aldosterona y cortisol; todos estos niveles plasmáticos se encuentran por debajo de las cifras normales. Las concentraciones de DOC y corticosterona deben estar por encima de lo normal (tabla 4). Si los niveles de estas últimas hormonas están por encima de los normales, acompañados de hipertensión e hipocaliemia, se debe confirmar el diagnóstico con ciertas pruebas, entre ellas el estudio genético ${ }^{(20)}$.

\section{Tumores productores de DOC}

Los tumores adrenales productores de DOC son muy raros y usualmente muy grandes y malignos ${ }^{(20,37)}$; se han descrito tumores productores de DOC benignos ${ }^{(20)}$.

\section{Presentación clínica}

Algunos de estos tumores son capaces de secretar andrógenos y estrógenos, por lo cual pueden producir virilización en mujeres y feminización en hombres. Como clásico, estos tumores crecen rápidamente, acompañados de marcada hipertensión asociada a la hipocaliemia y bajos niveles de aldosterona y renina ${ }^{(20)}$.

Se deben investigar los pacientes con hipertensión, hipocaliemia espontánea y niveles de aldosterona y renina bajos.

La presencia de niveles altos de DOC en plasma o de tetrahidrodesoxicorticosterona en orina, debido a un gran tumor adrenal detectado por TAC, confirman el diagnóstico ${ }^{(20)}$. 


\section{Resistencia primaria al cortisol}

Esta es una entidad familiar rara, caracterizada por resistencia al cortisol (glucocorticoides); típicamente los niveles plasmáticos de cortisol están elevados, sin que se presente un síndrome de Cushing ${ }^{(20)}$. Se explica este síndrome por defecto genético en los receptores de los glucocorticoides, lo que produce resistencia a las acciones del cortisol, a nivel periférico y central $^{(20)}$.

Este síndrome se caracteriza por hipocaliemia, alcalosis, hipertensión, incremento en las concentraciones plasmáticas de DOC y exceso en la secreción de andrógenos. La hipertensión y la hipocaliemia se deben conjuntamente al exceso de DOC y al aumento de la sensibilidad de los receptores de los mineralocorticoides al exceso de cortisol circulante ${ }^{(20)}$.

Se debe investigar especialmente niños portadores de hipertensión, hipocaliemia espontánea y niveles bajos de renina $\mathrm{y}$ aldosterona $^{(20)}$.

Como pruebas iniciales en el diagnóstico de esta entidad se deben medir en plasma, cortisol, DOC, 11-desoxicortisol, androstenediona, testosterona y dehidroepiandosterona sulfato; los niveles de estas hormonas en los casos positivos están por encima de los límites normales (tabla 4). Además, la medición de la excreción del cortisol urinario está elevada y los niveles de ACTH no están suprimidos ${ }^{(20)}$. Para confirmación del diagnóstico se realizan estudios de mutaciones genéticas.

\section{Síndrome de aparente exceso de mineralocorticoides. Deficiencia de la $11 \beta$-hidroxiesteroide deshidrogenasa}

Este síndrome se debe a disminución de la actividad de la enzima microsomal HSD11B2, que normalmente inactiva el cortisol en el riñón, al convertir el cortisol en cortisona ${ }^{(38)}$. El cortisol puede actuar como un potente mineralocorticoide cuando existe mutación de la HSD11B2, con disminución de su actividad o cuando su actividad es bloqueada, se presentan altos niveles de cortisol a nivel renal ${ }^{(20)}$.

La disminución de la actividad de la enzima HDS11B, ya sea genética o debida a la inhibición de la enzima por el ácido glycyrrhiza (el principio activo de la raíz del licorice). Las formas congénitas se transmiten en forma autosómica recesiva. Se han identificado alrededor de cincuenta casos descritos en el mundo ${ }^{(20)}$.

\section{Hallazgos clínicos}

Los casos con aparente exceso de mineralocorticoides presentan en la niñez hipertensión, hipocaliemia, peso bajo al nacer, disminución en el crecimiento, poliuria y polidipsia ${ }^{(39)}$.

La forma adquirida de aparente exceso de mineralocorticoides debida a la ingestión de la raíz del licorice o de carbenoxolona producen hipertensión e hipocaliemia; el caso se descubre por medio de la historia clínica ${ }^{(39)}$. Cuando se presenta hipersecreción masiva de cortisol en el síndrome de Cushing, originada en la secreción ectópica de ACTH, se puede producir hipocaliemia e hipertensión ${ }^{(40)}$.

Los clínicos deben investigar a los pacientes con aparente exceso de mineralocorticoides, ya sea congénito o adquirido; estos pacientes pueden presentar hipertensión, hipocaliemia, alcalosis metabólica y niveles bajos de renina y aldosterona y niveles normales de cortisol. Los clínicos pueden confirmar este diagnóstico con la demostración de una relación muy elevada de cortisol a cortisona en orina de 24 horas. Esto se debe a una disminución de la actividad de la enzima HSD11B2. La relación de cortisol a cortisona está elevada 10 veces $^{(39)}$.

\section{Síndrome de Liddle; transporte iónico renal anormal}

En 1963, Liddle descubrió un trastorno autosómico dominante renal, similar a un hiperaldosteronismo que incluía hipertensión, hipocaliemia e inapropiada caliuresis ${ }^{(40,41)}$. Se han informado alrededor de 30 familias con este síndrome ${ }^{(20)}$.

Los clínicos deben investigar a niños y adultos que presentan hipertensión, hipocaliemia espontánea y niveles bajos de renina y aldosterona. Una historia familiar de este síndrome sugiere aún más el diagnóstico de esta posibilidad.

Una vez que el clínico excluye los otros casos con presentación similar (tabla 4), se debe considerar tratar al paciente con amilorida o triantireno. Se puede distinguir el síndrome de Liddle del aparente exceso de mineralocorticoides, basado en la respuesta marcada de la hipertensión a la administración de amilorida o triamtereno en el síndrome de Liddle y no en los otros síndromes.

Se puede diferenciar el diagnóstico de síndrome de Liddle del aparente exceso de mineralocorticoides también por la ineficiencia terapéutica de la espirolactona y de la dexametasona, en presencia de una relación cortisona/cortisol en orina de 24 horas, normal ${ }^{(20)}$.

\section{Aldosteronismo secundario debido a hipertensión renovascular}

Hace más de 80 años se observó que la reducción del flujo sanguíneo a los riñones podía iniciar una hipertensión arterial denominada hipertensión renovascular. Después de varios años se demostró como causa de esta hipertensión al sistema renina-angiotensina-aldosterona ${ }^{(20)}$.

El hiperaldosteronismo secundario se caracteriza por niveles altos de aldosterona debido a la activación del eje renina-angiotensina II. Otras causas del hiperaldosteronismo secundario son el infarto renal, depleción del volumen debida o no a diuréticos, hipoperfusión renal originada en la falla cardiaca o renal, o raramente a hiperproducción de renina dependiente de un tumor renal yuxtaglomerular. El hiperal- 
dosteronismo secundario debido a depleción de volumen, a falla cardiaca o renal, no se asocia con hipertensión arterial, generalmente.

Aunque son frecuentes las estenosis renales por arterioesclerosis y las displasias fibromusculares, éstas pueden ser asintomáticas, sin hipertensión arterial; se desconoce la verdadera prevalencia de la hipertensión renovascular, pero se cree que en los centros de referencia de hipertensos al menos del 1\% al 5\% pueden tener hipertensión renovascular ${ }^{(20)}$.

\section{Presentación clínica}

La enfermedad renovascular oclusiva puede dar origen a un espectro de manifestaciones, que incluyen hipertensión renovascular, una forma acelerada de hipertensión maligna, daño cardiaco, congestión circulatoria (edema pulmonar) y, por último, daño renal irreversible.

La necesidad de estudiar y detectar las causas de la hipertensión renovascular en el momento actual ha disminuido por una razón, la terapia médica es altamente efectiva en el bloqueo del sistema renina-angiotensina-aldosterona, sin necesidad de cirugías endovasculares o de revascularizaciones ${ }^{(20)}$. Se puede sugerir que los clínicos solamente deben limitar el tamizaje en los pacientes en los cuales falla la terapia médica o presentan

Tabla 5. Hallazgos clínicos debidos a enfermedad renovascular

Generalmente la hipertensión arterial se inicia antes de los 30 años

Hipertensión acelerada, resistente, hipertensión maligna

Daño renal (elevación de la creatinina de más del 30\%), en respuesta a los hipotensores inhibidores ECA o inhibidores de los receptores de la angiotensina

Comienzo de la hipertensión después de los 50 años sugestiva de estenosis por arteriosclerosis de la arteria renal

Riñones asimétricos con una diferencia de más de $1,5 \mathrm{~cm}$ en el tamaño y por pérdida de la función renal sin causa aparente

Edema pulmonar inexplicado de comienzo agudo riesgos de terminar con daño renal o cardiaco irreversibles, si no se restablece la circulación local renal (tabla 5).

En la referencia 20 pueden revisarse los diferentes métodos imaginológicos, su sensibilidad y especificidad en el diagnóstico de la estenosis renal por arteriosclerosis o displasia renal, lo mismo que los estudios endocrinológicos para obtener un diagnóstico de precisión.

\section{Otras entidades endocrinas asociadas con hipertensión arterial}

Solo enumeraremos estos estados y no profundizaremos sobre ellos, por limitaciones de espacio; en la referencia 20 se detalla sobre la prevalencia, presentación clínica, cómo deben evaluarse, las pruebas diagnósticas y el tratamiento de las entidades anotadas. Estos estados son: síndrome de Cushing, el hipertiroidismo, el hipotiroidismo, la hipercalcemia, la acromegalia y la apnea obstructiva del sueño.

\section{Resumen}

La hipertensión arterial puede ser la primera presentación en al menos 15 entidades endocrinas. Un diagnóstico preciso temprano de una hipertensión endocrina brinda la posibilidad al internista o endocrinólogo de conseguir una cura por medio de cirugía o de obtener un control estricto de la entidad con una terapia farmacológica específica. La experiencia ha demostrado que los clínicos deben tener presente la presencia de hiperaldosteronismo primario en la mayoría de los casos de hipertensión arterial y de estar alerta sobre la presencia de un feocromocitoma o un paraganglioma o ciertos casos sospechosos de la entidad.

El estudio de los pacientes hipertensos con una entidad endocrina hace posible con frecuencia llegar a un diagnóstico de precisión en entidades en las cuales es difícil pensar, con base solamente el cuadro clínico solapado o inexistente o por su rareza, como el síndrome de aparente exceso de mineralocorticoides, la hiperplasia adrenal congénita, o la apnea obstructiva; haciendo posible el control de las complicaciones a largo plazo de la hipertensión. 


\section{Referencias}

1. Yoon SS, Ortega Y, Louis T. Recent trends in the prevalence of high blood pressure and its treatment and control, 1999-2008. NCH Data Brief. 2010; (48):1-8.

2. James PA, Oparil S, Cushman WC, et al. 2014 evidence-based for the manegment of high blood pressure in adults: report from the panel members appointed to Eight Join National Committel (JNC.8). JAMA 2014; 311 (5): 507520.

3. Fields LE, Burt VL, Cutler, et al. The burden of adult hypertension in the United States. 1999 to 2000: a rising tide. Hypertension Res. 2004;44(4): 398-404.

4. Rudnick KV, Sackett DL, Hirst S, et al. Hypertension in a family practice. Can Med Assoc J, 1977 17(5): 492-497.

5. Omura M, Saito J, Yamaguchi K, et al. Prospective study on the prevalence of secondary hypertension among hypertensive. Patents visiting a general clinic in Japan. Hypertension Res 2004; 27 (3): 193-202.

6. Gupta-Malhotra M, Banker A, Shetes, et al. Essential hypertension vs secondary hypertension among childrens. Am J Hipertension. 2015; 28 (1): 73-80.

7. Camelli S, Bobrie G, Postel-Viney E, et al. Prevalence of secondary hypertension in young hypertensive adults. J Hipertension. 2015; 33 (Suppl 1): c47.

8. O. Shea PM,Griffin TP, Fitzgibbon M, Hypertension: The role of biochemistry in the diagnosis and manegement.ClinChim Acta. 2017; 465:131-143.

9. Funder JW. Carey RM, Mantero F, et al.The manegement of primary aldosteronism: case detection, diagnosis and treatment in Endocrine Society Clinical Practice Guideline. J Clin Endocrinol Metab. 2016;101(5): 1889-1916.

10. Lender JW, Eisenhofer G,Monnelli M, et al. Phaechromocytoma. Lancet. 2005;366 (9486): 655-675.

11. Delellis R, Lloyd R, et al. Pathologic and genetics of tumours of endocrine organs. Lyon, France: IAAC Press. 2004.

12. Mansmann G, Lau J,Balk E, et al. The clinically inapparent adrenal mass: uptodate in diagnosis and management Endocr Rev.2004; 25 (2): 309-340.

13. Lo CY, Lam KY, Wat MS, et al. Adrenal pheocromocytoma remains frequently overlooked diagnosis . Am J Surg. 2000; 179 (3): 212-215.

14. Beard CM, Sheps SG, Kurland LT, et al. Ocurrence of preochromocytoma in Rochester, Minnesota, 1950 through 1979. Mayo Clin Proc. 1983;58 (12): 802-804.

15. Kimura N,Miura Y Nagatsu N, et al. Catecholamines synthesizing enzimes in 70 cases of functioning and no functioning and extradrenal paraganglioma. Virhows Arch A Pathol Anat Histopathol. 1992; 421(1): 25-32.

16. Eisenhofer G, Lenders JW, Golstein DS,et al. Pheochromocytoma catecholamines phenotypes and prediction of tumor size and localization by use of plasme metanephrines. Clin Chem. 2005;51(4): 735-744.

17. Eisenhofer G, Pack K, Huynh TT, et al. Catecholamic metabolic and and secretory phenotypes in phaeochromocytoma. Endocr Relat Cancer. 2010;18(1): 977-1111.

18. Eisenhofer G, Huynh TT, Elkahloun A, et al. Differential expression of the regulated catecholamines secretory pathway in different hereditary forms of pheochormocytoma. Am j Phisiol Endocrinol Metab. 2008; 295 (5): E1223-E1233.

19. Bravo EL, Tagle, R. Pheochomocytoma: of-the-art and future prospects. Endocr Rev. 2003; 24(4): 539-553.

20. Young F, Young Jr. Calhoun AD, et al- Screeming for endocrine hypertemsion: An Endocrine Society Scientific Statement. Endocr Rev. 2017; 38(2): 103122.

21. Young WF, Jr. Clinical practice. The incidentally discoverd adrenal mass. New Engl J Med. 2007; 356(6): 601-610.

22. Lender JW, Duh QY, Eisenhofer G, et al. Endocrine Society Clinical. Pheo- chromocytoma and paraglanglioma: Endocrine Clinical Society. Practice guideline. J Clin Endocrinol Metab. 2014: 99(6): 1915-1942.

23. Eisenhofer G, Lender JW, Siegert G, et al. Plasma methoxy tyramine a novel biomarker of metastasic pheochomocytoma and paraganglioma in relation to established size factor of tumor rize localization and SDHB mutation status. Eur J Cancer. 2012; 48(11): 1739-1749.

24. Algeciras-Schimmich A, Preissner CM, et al. Plasma chormogranin A or urine factioned metanephrines follow-up testing improves the diagnost accuracy of plasma fractioned metanephrines for pheocromocytoma, J Clin Endocrinol Metab. 2008: 93(1): 91-95.

25. Eisenhofer G, Golstein DS, Walther MM, et al. Biochemical diagnosis of pheocromocytoma: how to distinguish true-from false-positive test result. J Clin Endocrinol Metab. 2003; 88(6). 2656-2666.

26. Conn JW, Presidential address. I. Painting II. Primary aldosteronism, a new clinical-syndrome. J Lab Clin Med. 1995; 45(1): 3-17.

27. Rossi GP, Bernini G, Caliumi C, et al. Study Investigator. A prospective of the prevalence of primary aldosteronism in 1.125 hipertensive patients. J Am Coll Cardiol. 2006: 48(11): 2293- 2300.

28. Conn JW, Knopf RF, Nesbit RM. Clinical characteristics of primary aldosteronism from ananalysis of 145 cases. AM J Surg. 1964; 107: 159-172.

29. Mulatero P, Stowasser M, Loh KC, et al. Increased diagnosis of primary aldosternonism, in cluding surgical correctable forms, in centers from five continents. J Clin Endocrinol Metab. 2004; 89(3): 1045-1050.

30. Young WF, Jr; Klee GG. Primary aldosteronism .Diagnostic evaluation. Endocrinol Metab Clin North Am. 1988; 17(2): 367-395.

31. Ono Y, Iwakura Y, Morimoto R, et al. Os 35-01 the prevalence of sleep apnea sindrome in primary aldosteronism. J Hypertens. 2016; 34 (Suppl 1ISH2016 Abstrat Book: e399.

32. Cellen $0,0^{`}$ Brien MJ, Beazley RM, et al. Factors influencing outcome of surgery for primary aldosteronism. Arch Surg. 1996; 131(6): 646-650.

33. Rutherford JC, Tayor WL Stowasser M, et al. Success of surgery for primary aldosteronism judged by residual autonomous aldosterone production. World J Surg. 1998;22 (12): 1243-1245.

34. Stowasser M, Gordon. Primary aldosteronism- careful investigation is essential and rewarding. Moll Cell Endocrinol. 2004; 217(1-2): 33-39.

35. Speiser PW, Azziz R, Baskin LS, et al. Congesnital adrenal hiperplasia due to steroid 21- hyrdoxylase deficiency: an Endocrine Society clinical Practical Guideline. J Clin Encodrinol Metab. 2010; 95 (9): 4133-4160.

36. Kim YM, Kang M, Choi JM, et al. Areview of the literature on common YP17A1 mutations in adults with 17-hydroxilase/17,20, lyase deficiency, a case series of such mutations among koreans and functional characteristics of a novel mutation. Metabolism 2014; 63(1): 42-49.

37. Ishikawa SE, Saito T, Kaneko K, et al. Hipermineracorticism without elevation of plasma aldosterone; deoxicorticosterone-producing adrenal adenoma and hiperplasia. Clin Endocrinol (Oxford). 1988;29 (4): 367.

38. Chapman K, Holmes M, Seckl J. 11 beta- Hidroxiesteroid dehidrogenase; intracellar gatekeepers of tissue glucorticoid action. Phisiol Rev. 2013; 93(3): 1139-1206.

39. Stewart PM, Krozowski ZS, Gupta A, et al. Hypertension in the sysdrome of apparent mineralocorticoid excess due to mutation of the 11beta hidroxiesteroid deshidrogenasa type 2 gene. Lancet 1996: 347(8994): 88-91.

40. Nieman L, Biller BM, Findling W, et al. The diagnosis of Cushing syndrome: an Endocrine Society Clinical Practice Guideline.J Clin Endocrinol Metab. 2008; 93 (5): 1526-1540.

41. Liddle G W. A familial renal disorder simulating primary aldosteronism but with negligible aldosterone secretion. Trans Assoc An Physicians. 1963; 76:199-213. 